\title{
¿QUIÉN PARTICIPA? UNA APROXIMACIÓN A LOS Determinantes de la PARTICIPACIÓN POLÍtica en ChILE
}

\author{
Carolina Segovia (Chile)
}

\section{Introducción}

Cualquiera sea la definición de democracia que uno utilice, la participación política de sus ciudadanos aparece como un elemento central a considerar. En efecto, la manifestación de preferencias y demandas ciudadanas a los gobernantes es un componente importante para un buen funcionamiento democrático (ver, por ejemplo, Diamond y Morlino, 2005). Una baja participación, entonces, puede traer, entre otras, las siguientes consecuencias para un sistema democrático: baja en la legitimidad electoral de las autoridades políticas; menor representación de grupos específicos dentro de la población de un país y la ausencia de temas de políticas públicas relevantes a esos grupos; sesgos o inequidades en la expresión de preferencias (Rosenstone y Hansen, 1993; Altman y PérezLiñán, 2002; Verba, Schlozman y Brady, 1995).

Utilizando datos provenientes del estudio "Auditoría a la Democracia en Chile", este trabajo es un análisis preliminar de los niveles de participación política de los

Socióloga, P. Universidad Católica de Chile y Ph.D. en Ciencia Política, University of Michigan. Actualmente se desempeña como coordinadora del programa de opinión pública del Centro de Estudios Públicos (CEP). E-mail: csegovia@cepchile.cl. 
chilenos, así como una aproximación a los determinantes individuales de dicha participación.

El proyecto "Auditoría a la Democracia en Chile" está siendo realizado por un consorcio de Centros de Estudios chilenos (CEP, CIEPLAN, Libertad y Desarrollo, y ProyectAmérica) en conjunto con PNUD-Chile, desde el año 2009. ${ }^{2}$ Este proyecto tiene, como objetivo central, la realización de una evaluación de la calidad de la democracia chilena. Dentro de las actividades contempladas en el proyecto, se realizó una encuesta nacional a 1.522 personas mayores de 18 años, residentes a lo largo de todo Chile, considerando zonas urbanas y rurales, entre el 25 de septiembre y el 25 de octubre de 2010. Esta encuesta incluyó preguntas asociadas a aspectos variados de la democracia como derechos y deberes ciudadanos, evaluación del Congreso, partidos políticos y Poder Judicial y participación política entre otros, y contó con el financiamiento de IDEA-Internacional. ${ }^{3}$ En lo que sigue, reportaremos resultados obtenidos en esta encuesta respecto de los niveles de participación política declarados por los ciudadanos, así como resultados preliminares en torno a los determinantes individuales de dicha participación.

2 Las opiniones aquí presentadas y discutidas no representan, sin embargo, a las instituciones o personas que han participado o colaborado en este proyecto.

3 El cuestionario, características del diseño metodológico, resultados y bases de datos pueden ser obtenidos en www.auditoriaalademocracia. cl y en www.cepchile.cl. 


\section{Participación política en Chile: baja y poco relevante para los ciudadanos}

En la elección presidencial de diciembre de 2009, 7.264.136 personas concurrieron a las urnas a votar. Esto representa el $87,7 \%$ del padrón electoral chileno. ${ }^{4}$ A simple vista, estos números de participación parecen altos, particularmente si uno los compara con los niveles de participación de otras democracias más consolidadas (Blais, 2007). Esta tasa de participación, sin embargo, esconde un fenómeno que se ha desarrollado a partir de 1993 en adelante, que indica que la participación electoral ha caído en forma sistemática en Chile. En efecto, tal como se observa en la figura 1, aun cuando una mayoría significativa de personas inscriptas vota en las elecciones (VE / I), el número de inscriptos en los registros electorales se ha mantenido bastante estable desde 1988, llevando a una caída en el número de personas que votan en comparación con la población en edad de votar (VE / PEV). Si hacia 1989 el 84\% de la población en edad de votar (PEV) votó, hacia 2009, sólo el $60 \%$ de la PEV lo hizo. ${ }^{5}$

Para la elección presidencial y parlamentaria de 2009, el padrón electoral estaba compuesto por 8.285.186 de personas (Fuente: Servel).

El voto en Chile es obligatorio. Sin embargo, para poder votar los ciudadanos deben realizar el trámite de inscripción en el registro electoral, el cual es voluntario. Es necesario destacar que en el año 2009 fue aprobada una reforma constitucional que define al voto como voluntario. Para que la reforma entre en vigencia, sin embargo, se requiere la aprobación de la ley que permita el cambio a un sistema con inscripción automática y voto voluntario. A la fecha en que se escribe este artículo, dicha ley se encuentra en discusión en el Parlamento. 
Figura 1. Participación en elecciones presidenciales y parlamentarias 1989-2009

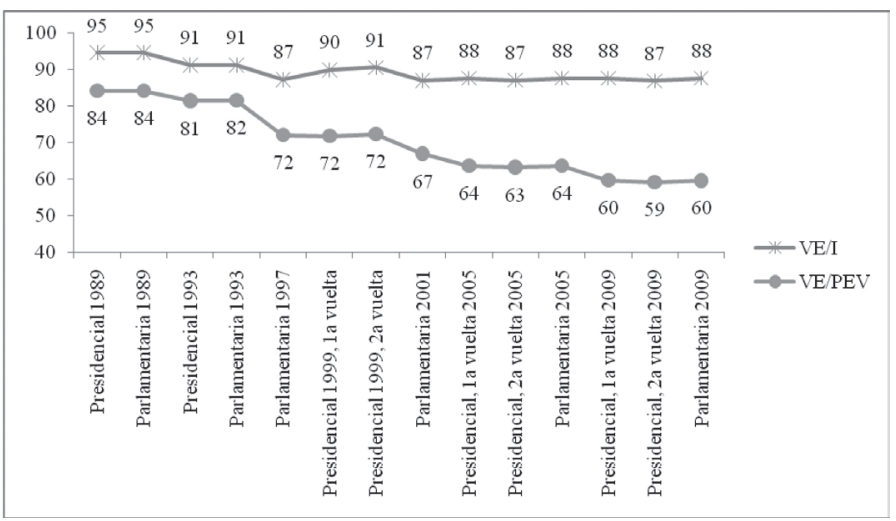

Fuente: Elaboración propia con datos obtenidos en www.servel.cl, www.elecciones.gov.cl y www.ine.cl

Si las personas no participan en las elecciones, eso no significa, a priori, que ellas no realicen ningún tipo de actividad política. La participación puede adquirir distintas formas que, como señalan Verba et al. (1995) presentan variados tipos de ventajas y requisitos para su realización por parte de los ciudadanos. Brady (2003), por ejemplo, señala que dentro de aquellas actividades que buscan influir en la política se puede distinguir entre actividades electorales y no electorales. Las actividades electorales incluyen el voto, pero también otras como participar en campañas, donación de dinero para partidos o candidatos, etc. Dentro de las actividades no electorales distingue entre aquellas formas de participación convencionales (como la pertenencia a organizaciones secundarias) y aquellas no convencionales: firmar peticiones, asistir a marchas, participar en huelgas, etc. Es posible, entonces, que hubiese un proceso de desencanto con el voto como forma de expresión de intereses 
y demandas, pero una ciudadanía activa en otros niveles o formas de participación. En otras palabras, dado el amplio espectro de formas de participación, las personas pueden dejar de votar pero no necesariamente dejar de participar.

Resultados obtenidos en Chile, sin embargo, indican que esto no ocurre. Los niveles de participación son bajos en los distintos modos de participación que las personas pueden utilizar. La tabla 1 presenta los porcentajes de personas que señalan haber participado en alguna de las actividades indicadas. Tal como se puede apreciar, salvo la inscripción en los registros electorales, la participación en actividades políticas es baja, una anomalía más de la norma en el caso de los chilenos. En efecto, un $73,3 \%$ señala no haber realizado ninguna de las actividades políticas consideradas, y alrededor de un $14 \%$ señala haber realizado 2 o más actividades. La participación en organizaciones secundarias, incluso en aquellas más alejadas de la actividad política, es también baja: poca gente en Chile participa en actividades políticas o en organizaciones donde interactúe o trabaje junto a otros. Un $64 \%$ de los entrevistados señala no participar en ningún tipo de organización, y un $23 \%$ señala que participa en una organización.

Tabla 1. Participación política, 2010

\begin{tabular}{|c|c|}
\hline & $\%$ \\
\hline Inscrito en los Registros Electorales (\% Sí) & $68,6 \%$ \\
\hline \multicolumn{2}{|l|}{ Actividades Políticas (\% sí) } \\
\hline Firmado alguna petición & $10,3 \%$ \\
\hline Asistido a una marcha o manifestación política & $12,0 \%$ \\
\hline Donado dinero o recolectado fondos para una actividad social o política & $15,2 \%$ \\
\hline $\begin{array}{l}\text { Contactado o figurado en los medios de comunicación para expresar } \\
\text { su opinión }\end{array}$ & $5,3 \%$ \\
\hline Participado en un foro político o en un grupo de discusiones en Internet & $4,7 \%$ \\
\hline Participado en una huelga & $7,7 \%$ \\
\hline Trabajado en una campaña electoral & $5,5 \%$ \\
\hline
\end{tabular}




\begin{tabular}{|c|c|}
\hline \multicolumn{2}{|c|}{ Pertenencia a Organizaciones Secundarias (\% Pertenece) } \\
\hline Partido Político & $3,2 \%$ \\
\hline Sindicato & $5,0 \%$ \\
\hline Asociación profesional o gremial & $3,9 \%$ \\
\hline Asociación empresarial & $1,7 \%$ \\
\hline Organización religiosa & $18,4 \%$ \\
\hline Grupo Cultural & $6,5 \%$ \\
\hline Grupo deportivo & $10 \%$ \\
\hline Fundación o grupo de beneficencia & $4,1 \%$ \\
\hline Otra asociación voluntaria & $6,4 \%$ \\
\hline
\end{tabular}

Fuente: Encuesta Auditoría a la Democracia en Chile, 2010.

Los chilenos, entonces, declaran un bajo nivel de participación y asociación. Al mismo tiempo, y tal vez por esta misma razón, la participación no resulta bien valorada por los chilenos. En efecto, resultados del mismo estudio indican que menos del $50 \%$ de los entrevistados consideran que es "muy importante" votar siempre en las elecciones, y apenas un $18 \%$ considera que es muy importante para un buen ciudadano ser activo en organizaciones sociales y políticas. ${ }^{6}$

En síntesis, entonces, hacia fines de 2010, los niveles de participación y de valoración de la participación son bajos, manteniendo una tendencia que ha sido observada con anterioridad respecto del escaso nivel de involucramiento de los chilenos en su sistema político (De la Maza, 2010). ${ }^{7}$

$6 \quad$ Más de un $50 \%$ de los entrevistados, por otra parte, señala que para un buen ciudadano es muy importante "siempre obedecer las leyes" y "ayudar a las personas en Chile que están en peores condiciones que uno".

Datos obtenidos por proyectos de opinión pública comparados como LAPOP, Latinobarómetro, y el Estudio Mundial de Valores muestran también que la participación política en Chile es baja comparada con aquella observada en otros países de la región. 


\section{3. ¿Quién participa? Determinantes individuales de la participación política en Chile}

Existe una vasta literatura respecto de los determinantes individuales de la participación política que, por razones de espacio, no podemos discutir en este trabajo. Sin embargo, es necesario mencionar brevemente algunos de los elementos centrales. Primero, la investigación ha mostrado que dado el amplio espectro de formas de participación a las que los ciudadanos tienen acceso, el efecto que tienen los distintos factores a considerar puede variar, tanto en la dirección del efecto, como en su relevancia (Brady, 2003; Verba et al., 1995). Por ejemplo, ciertas formas de participación pueden ser más habituales o probables entre los más jóvenes y otras entre las personas de mayor edad. $\mathrm{O}$, si se considera el efecto del nivel de ingreso de los individuos, éste puede ser más relevante para explicar donaciones monetarias a las campañas que para explicar la firma de una petición. No podemos esperar, por lo tanto, que los mismos factores afecten los niveles de participación en distintas actividades de la misma manera.

En segundo lugar, la investigación ha centrado su atención en distintos tipos de variables que pueden afectar la participación política. A nivel individual, es decir, cuando se quieren explorar diferencias entre los individuos que participan y aquellos que no, es posible distinguir en la literatura cuatro tipos de factores relevantes: características demográficas; nivel o estatus socioeconómico; actitudes; y factores políticos (Brady, 2003; Rosenstone y Hansen, 1993; Verba et al., 1995; Klesner, 2007). El foco de este trabajo estará en una primera aproximación al efecto de estos factores individuales.

Tendremos en cuenta, entonces, los efectos que las siguientes variables puedan tener en las distintas formas de participación consideradas en la sección anterior. Primero, dentro de los factores demográficos incluimos la edad y el sexo de las personas. La literatura ha mostrado que el efecto 
de edad, y en cierta medida también el del sexo, son relevantes para explicar la participación, aun cuando estos efectos varían de acuerdo al tipo de participación. Así, por ejemplo, la participación electoral sería mayor entre las personas de mayor edad, mientras que la participación en actividades no convencionales sería mayor entre los más jóvenes (Wattenberg, 2006).

En segundo lugar, como medida de estatus socioeconómico incluimos el nivel educacional, medido a través de los años de escolaridad de los individuos. La hipótesis, en este caso, es que la participación debiera ser mayor entre aquellos con mayores niveles educacionales (Verba et al., 1995; Rosenstone y Hansen, 1993; Klesner, 2007; Norris, 2002).

Como variable actitudinal incluimos el nivel de interés en la política, que tal como se ha visto tiende a aumentar los niveles de participación (Brady, 2003). Finalmente, como factor político incluimos la identificación de las personas con posiciones políticas. En este caso, la literatura también ha mostrado que su efecto varía dependiendo del contexto y del tipo de participación (Brady, 2003).

Comenzaremos el análisis con una revisión de las relaciones bivariadas entre estos factores y nuestras medidas de participación. La tabla 2 presenta los porcentajes de participación observados según sexo, edad, años de escolaridad de los entrevistados, interés en la política y posición política con la que se identifican. Las variables dependientes corresponden a la inscripción en los registros electorales, al número de actividades políticas realizadas y al número de organizaciones a las que pertenecen los entrevistados.

Estos resultados muestran, por una parte, y confirmando lo señalado antes, que la participación, ya sea en organizaciones secundarias o en actividades no convencionales, es baja para cada uno de los grupos estudiados. Aun cuando existen diferencias, no existen grupos específicos que presenten porcentajes mayoritarios de participación entre sus miembros. Los resultados también muestran, en segundo 
lugar, que los niveles de participación en cada unos de estos tres tipos considerados varían en forma específica, indicando la presencia de preferencias específicas de acuerdo al sexo, edad, nivel educacional, posición política y nivel de interés en la política. Veamos estos resultados en mayor detalle.

Tabla 2. Niveles de participación según sexo, edad y años de escolaridad

\begin{tabular}{|c|c|c|c|c|c|c|}
\hline & \multicolumn{2}{|c|}{$\begin{array}{l}\text { Está inscripto en los } \\
\text { registros electorales }\end{array}$} & \multicolumn{2}{|c|}{$\begin{array}{l}\text { Pertenece a organiza- } \\
\text { ciones secundarias }\end{array}$} & \multicolumn{2}{|c|}{$\begin{array}{l}\text { Participación en } \\
\text { actividades políticas }\end{array}$} \\
\hline & Sí & No & Ninguna & 1 o más & Ninguna & 1 o más \\
\hline \multicolumn{7}{|c|}{ Sexo } \\
\hline Mujer & 69,0 & 31,0 & 68,2 & 31,8 & 77,0 & 23,0 \\
\hline Hombre & 68,6 & 31,4 & 59,9 & 40,1 & 69,5 & 30,5 \\
\hline \multicolumn{7}{|c|}{ Edad } \\
\hline $18-24$ & 17,9 & 82,1 & 65,2 & 34,8 & 66,4 & 33,6 \\
\hline $25-34$ & 45,9 & 54,4 & 68,4 & 31,6 & 67,5 & 32,5 \\
\hline $35-54$ & 84,9 & 15,1 & 63,2 & 36,8 & 76,6 & 23,4 \\
\hline 55 y más & 97,0 & 3,0 & 61,6 & 38,4 & 77,8 & 22,2 \\
\hline \multicolumn{7}{|c|}{ Años de escolaridad } \\
\hline $0-3$ & 79,8 & 20,2 & 66,7 & 33,3 & 94,0 & 6,0 \\
\hline $4-8$ & 77,1 & 22,9 & 71,8 & 28,2 & 84,1 & 15,9 \\
\hline $9-12$ & 62,6 & 37,4 & 64,6 & 35,4 & 75,6 & 24,4 \\
\hline 13 y más & 68,9 & 31,1 & 56,5 & 43,5 & 57,1 & 42,9 \\
\hline \multicolumn{7}{|c|}{ Interés en la política } \\
\hline $\begin{array}{l}\text { Poco / nada } \\
\text { interesado }\end{array}$ & 67,6 & 32,4 & 65,3 & 34,7 & 75,7 & 24,3 \\
\hline $\begin{array}{l}\text { Bastante/muy } \\
\text { interesado }\end{array}$ & 81,0 & 19,0 & 49,7 & 50,3 & 50,0 & 50,0 \\
\hline \multicolumn{7}{|c|}{ Posición Política } \\
\hline Derecha & 77,1 & 22,9 & 57,1 & 42,9 & 66,9 & 33,1 \\
\hline Centro & 72,3 & 27,7 & 60,5 & 39,5 & 77,7 & 22,3 \\
\hline Izquierda & 68,4 & 31,6 & 55,9 & 44,1 & 56,1 & 43,9 \\
\hline Ninguna & 65,0 & 35,0 & 70,9 & 29,1 & 81,0 & 19,0 \\
\hline
\end{tabular}

Fuente: Elaboración propia con datos de Encuesta Auditoría a la Democracia en Chile, 2010. 
Respecto de la inscripción en los registros electorales, los datos muestran que ella se produce de forma pareja entre mujeres y varones. En cambio, la inscripción parece asociada, de acuerdo a los datos en la tabla 2 , a variaciones respecto del nivel educacional y de la edad de las personas. Si consideramos años de escolaridad, observamos que la inscripción es mayor entre aquellos con menores niveles educacionales (hasta 8 años de escolaridad) que entre aquellos entrevistados con mayores niveles educacionales, y la menor tasa de inscripción se observa entre quienes tienen entre 9 y 12 años de escolaridad. Este resultado va contra lo esperado, pero, como veremos más adelante, es modificado en el análisis multivariado.

Los datos por edad son aquellos donde emerge el patrón más claro de diferencia: entre los jóvenes de entre 18 y 24 años de edad, la inscripción alcanza sólo al 18\%, mientras que en aquellos mayores de 55 años de edad, un $97 \%$ señala estar inscrito. Esto confirma lo señalado al comienzo de este artículo respecto de la estabilidad del padrón electoral chileno (muy pocos nuevos votantes se incorporan cada año). Este resultado también muestra el gran sesgo respecto del voto en Chile: las preferencias y demandas de los más jóvenes apenas pueden ser expresadas en las elecciones, dado el bajo porcentaje de este grupo que se inscribe y participa en los procesos eleccionarios.

La inscripción en el registro electoral, finalmente, aparece relacionada con fuerza al nivel de interés en la política, alcanzando un $81 \%$ de inscriptos entre aquellos que señalan tener mucho o bastante interés en la política, versus un $68 \%$ entre quienes manifiestan poco o nada de interés. Los resultados respecto de la identificación política no muestran diferencias muy importantes entre aquellos que se identifican con la derecha, el centro, o la izquierda, pero sí es relativamente menor el porcentaje de inscripción 
entre aquellos que no se identifican con ninguno de esos sectores o que no manifiestan preferencia política.

Respecto del capital social o, más específicamente, de la participación en organizaciones secundarias, los resultados muestran tres elementos importantes. Primero, que la participación en grupos u organizaciones es significativamente mayor entre varones que entre mujeres. Segundo, que no existen diferencias importantes por grupos etarios, con porcentajes de personas que dicen pertenecer a una o más organizaciones que fluctúan en torno a un tercio de los entrevistados en cada grupo. Por otra parte, la participación en este tipo de organizaciones es muy superior entre aquellas personas que han completado su educación secundaria y tienen 13 o más años de estudios aprobados. Aquellos con menos años de escolaridad presentan niveles similares de pertenencia.

Finalmente, la pertenencia a organizaciones secundarias, aun cuando ellas no estén relacionadas de forma directa con la actividad política, aumenta de manera estadísticamente significativa entre aquellos que expresan interés por la política, y disminuye de un modo importante también entre aquellos que no se identifican o no manifiestan preferencias políticas.

Respecto de la participación en otras actividades políticas, los resultados muestran diferencias relevantes para las tres variables aquí consideradas. En efecto, la participación es superior entre los varones, entre quienes tienen hasta 34 años de edad, y entre las personas con mayores niveles educacionales. Particularmente importante parece ser el efecto del nivel de escolaridad en estos tipos de participación, donde el porcentaje de realización de al menos una de estas actividades aumenta desde un $6 \%$ para aquellos que tienen entre 0 y 3 años de escolaridad, hasta un $43 \%$ entre aquellos que tienen 13 o más años de escolaridad. 
Al igual que en los casos anteriores, la participación en este tipo de actividades también aparece positivamente relacionada con el nivel de interés por la política expresado por los entrevistados, y es mayor entre quienes se identifican con la derecha o con la izquierda -sobre todo en este último caso-, mientras que es considerablemente menor entre aquellos que se identifican con el centro político o que no manifiestan preferencias específicas.

Para terminar este análisis, parece necesario evaluar el efecto que cada uno de estos factores tiene en los niveles de participación al controlar por el efecto de las demás variables. Con este fin, la tabla 3 presenta los resultados de análisis de regresión logística donde incluimos todas las variables consideradas antes, para cada una de las tres formas de participación analizadas en este trabajo.

Tabla 3. Análisis de regresión logística

\begin{tabular}{|c|c|c|c|c|c|c|c|c|c|}
\hline & \multicolumn{3}{|c|}{$\begin{array}{l}\text { Está inscripto en los } \\
\text { registros electorales }\end{array}$} & \multicolumn{3}{|c|}{$\begin{array}{c}\text { Pertenece a } \\
\text { organizaciones } \\
\text { secundarias }\end{array}$} & \multicolumn{3}{|c|}{$\begin{array}{l}\text { Participación en } \\
\text { actividades políticas }\end{array}$} \\
\hline & B & Sig. & $\operatorname{Exp}(B)$ & B & Sig. & $\operatorname{Exp}(B)$ & B & Sig. & $\operatorname{Exp}(B)$ \\
\hline \multicolumn{10}{|c|}{ Sexo } \\
\hline Hombre & 0.062 & 0.672 & 1.064 & 0.275 & 0.013 & 1.317 & 0.253 & 0.043 & 1.287 \\
\hline \multicolumn{10}{|c|}{ Edad } \\
\hline $18-24$ & -5.464 & 0.000 & 0.004 & -0.363 & 0.055 & 0.696 & 0.282 & 0.170 & 1.326 \\
\hline $25-34$ & -4.169 & 0.000 & 0.015 & -0.521 & 0.003 & 0.594 & 0.146 & 0.454 & 1.157 \\
\hline $35-54$ & -2.077 & 0.000 & 0.125 & -0.168 & 0.256 & 0.845 & -0.167 & 0.337 & 0.846 \\
\hline \multicolumn{10}{|c|}{ Escolaridad } \\
\hline 4-8 años & 1.074 & 0.007 & 2.926 & -0.072 & 0.792 & 0.931 & 1.037 & 0.031 & 2.821 \\
\hline $9-12$ & 1.214 & 0.002 & 3.367 & 0.279 & 0.293 & 1.322 & 1.449 & 0.002 & 4.260 \\
\hline 13 y más & 1.580 & 0.000 & 4.854 & 0.506 & 0.065 & 1.659 & 2.077 & 0.000 & 7.984 \\
\hline \multicolumn{10}{|c|}{ Interés en la política } \\
\hline $\begin{array}{l}\text { Mucho / } \\
\text { Bastante }\end{array}$ & 0.785 & 0.002 & 2.193 & 0.346 & 0.042 & 1.414 & 0.686 & 0.000 & 1.986 \\
\hline
\end{tabular}




\begin{tabular}{|c|c|c|c|c|c|c|c|c|c|}
\hline & \multicolumn{3}{|c|}{$\begin{array}{l}\text { Está inscripto en los } \\
\text { registros electorales }\end{array}$} & \multicolumn{3}{|c|}{$\begin{array}{c}\text { Pertenece a } \\
\text { organizaciones } \\
\text { secundarias }\end{array}$} & \multicolumn{3}{|c|}{$\begin{array}{c}\text { Participación en } \\
\text { actividades políticas }\end{array}$} \\
\hline & $B$ & Sig. & $\operatorname{Exp}(B)$ & $B$ & Sig. & $\operatorname{Exp}(B)$ & $B$ & Sig. & $\operatorname{Exp}(B)$ \\
\hline \multicolumn{10}{|c|}{ Posición política } \\
\hline Derecha & 0.566 & 0.009 & 1.761 & 0.408 & 0.008 & 1.504 & 0.454 & 0.008 & 1.574 \\
\hline Centro & 0.370 & 0.094 & 1.447 & 0.297 & 0.072 & 1.346 & -0.034 & 0.863 & 0.967 \\
\hline Izquierda & 0.378 & 0.063 & 1.460 & 0.438 & 0.004 & 1.549 & 0.836 & 0.000 & 2.307 \\
\hline Constante & 2.287 & 0.000 & 9.847 & -0.967 & 0.000 & 0.380 & -3.019 & 0.000 & 0.049 \\
\hline $\begin{array}{l}\text { R2 de Cox y } \\
\text { Snell }\end{array}$ & \multicolumn{3}{|c|}{0.350} & \multicolumn{3}{|c|}{0.038} & \multicolumn{3}{|c|}{0.107} \\
\hline $\begin{array}{c}\text { R2 de } \\
\text { Nagelkerke }\end{array}$ & \multicolumn{3}{|c|}{0.493} & \multicolumn{3}{|c|}{0.052} & \multicolumn{3}{|c|}{0.155} \\
\hline $\begin{array}{l}\text { Porcentaje } \\
\text { pronostica- } \\
\text { do correcto }\end{array}$ & \multicolumn{3}{|c|}{82,5} & \multicolumn{3}{|c|}{65,3} & \multicolumn{3}{|c|}{75,4} \\
\hline
\end{tabular}

Fuente: Elaboración propia con datos de Encuesta Auditoría a la Democracia en Chile, 2010.

¿Qué nos indican estos resultados? Primero, el ser mujer disminuye el nivel de participación, ceteris paribus, tanto en actividades políticas no convencionales o de protesta como en la pertenencia a organizaciones secundarias. El sexo de las personas no parece relevante, sin embargo, en el caso de la inscripción en los registros electorales, tal como había sido señalado con anterioridad.

La edad de las personas, por su parte, tiene efectos variados dependiendo del tipo de participación que observemos. En efecto, los resultados muestran que la participación en actividades de protesta aumenta de manera estadísticamente significativa al controlar por otros factores entre los más jóvenes, y disminuye fuertemente 
respecto de la inscripción en los registros electorales. En otras palabras, y al controlar por otras variables, los jóvenes presentan una mayor participación en actividades no convencionales y una menor inscripción en los registros electorales (que redunda en una menor participación en las elecciones).

El efecto de los años de escolaridad, ceteris paribus, es bastante más parejo en los distintos modos de participación. Los resultados indican que las distintas formas de participación aumentan entre aquellos con mayores niveles educacionales, siendo este efecto particularmente importante al considerar las personas con 13 o más años de estudios aprobados. El interés en la política también está asociado de manera positiva a la participación, siendo estadísticamente significativo este efecto para los tres tipos de participación considerados.

Finalmente, los resultados por posición política, controlando por los otros factores, muestran que la participación es mayor en Chile, en general, entre aquellos que se identifican con alguna posición política (versus aquellos que no se identifican, la categoría base para el análisis). Al mismo tiempo, identificarse con la derecha o con la izquierda tiene un mayor efecto sobre la participación que aquellos que se identifican con el centro. Por último, es interesante destacar que el identificarse con la "derecha" tiene un efecto más importante sobre la probabilidad de inscribirse en los registros electorales, y que identificarse con la "izquierda" tiene un mayor efecto, ceteris paribus, sobre la probabilidad de participar en otras actividades políticas. 


\section{Conclusiones}

Los resultados presentados en este trabajo, respecto de los niveles de participación política y sus determinantes individuales en Chile, aunque preliminares, nos permiten realizar algunos comentarios a modo de conclusión.

Primero, los datos muestran que existe en Chile un bajo nivel de participación política, lo que sumado a la caída en la participación electoral muestra a una ciudadanía despolitizada y con escaso acceso a la toma de decisiones. No tenemos aquí, sin embargo, datos que nos permitan evaluar la evolución en el tiempo de la participación no electoral. Si ella hubiese aumentado -al mismo tiempo que caído la inscripción y el voto-, entonces indicaría un cambio en las estrategias preferidas por las personas para expresar su opinión. Más investigación se requiere, sin embargo, para evaluar esta posibilidad.

En segundo lugar, los resultados presentados en este trabajo tienden a confirmar la idea de que los distintos factores analizados se relacionan en forma distinta dependiendo del tipo de participación que se considere en el análisis. Particularmente, las variaciones en la edad de las personas, para el caso chileno, puede tanto aumentar como disminuir -o no afectar de manera estadísticamente significativa- la probabilidad de participación. Respecto de las otras variables aquí consideradas, ellas tienden a presentar un mayor o menor poder explicativo dependiendo del tipo de participación.

Estos resultados, finalmente, plantean una serie de preguntas que deberán ser abordadas en el futuro. Primero, parece relevante la exploración sobre la variación en el tiempo en los niveles de participación no electoral. Esto permitiría abordar la pregunta respecto de cambios en las estrategias usadas por los ciudadanos para la expresión de sus preferencias. Segundo, es necesario abordar la pregunta 
por la estructura de las instituciones o grupos que promueven la participación política. La presencia o ausencia de movilización política, así como las características que ellas adoptan, pueden ser un importante factor para explicar la baja participación observada. En conclusión, una exploración respecto de los efectos que estos bajos niveles de participación tienen en la calidad de la democracia chilena es una tarea pendiente.

\section{Bibliografía}

Altman, David y Pérez-Liñán, Aníbal (2002), "Assessing the Quality of Democracy: Freedom, Competitiveness and Participation in Eighteen Latin American Countries", Democratization, 9 (2), pp. 85-100.

Blais, André (2007), "Turnout in Elections", en Dalton, Russell J. y Klingemann, Hans-Dieter, The Oxford Handbook of Political Behavior, Oxford, Oxford University Press. Brady, Henry E. (2003), An Analytical Perspective on Participatory Inequality and Income Inequality, a paper for the Russell Sage Foundation Project on the "Social Dimensions of Inequality.

De la Maza, Gonzalo (2010), "La disputa por la participación en la democracia elitista chilena", Latin American Research Review, núm. 45, pp. 274-297.

Diamond, Larry y Morlino, Leonardo (eds.) (2005), Assessing the Quality of Democracy, Baltimore, The Johns Hopkins University Press.

Klesner, Joseph (2007), "Social Capital and Political Participation in Latin America. Evidence from Argentina, Chile, Mexico and Peru", Latin American Research Review, 42 (2), pp. 1-32. 
Norris, Pippa (2002), Democratic Phoenix. Reinventing Political Activism, Cambridge, Cambridge University Press.

Rosenstone, Steven J. y Hansen, John Mark (1993), Mobilization, Participation, and Democracy in America, Nueva York, Macmillan.

Verba, Sydney; Schlozman, Kay L.; y Brady, Henry (1995), Voice and Equality. Civic Voluntarism in American Politics, Cambridge, Harvard University Press.

Wattenberg, Martin P. (2006), Is Voting for Young People?, Longman. 
